\title{
Catalizadores para la Reacción de Electrooxidación de Amoniaco: Estudio con Diferentes Concentraciones de $\mathrm{NH}_{4} \mathrm{OH}$
}

\section{Catalysts for the Electrooxidation Ammonia Reaction: Analysis with different $\mathrm{NH}_{4} \mathrm{OH}$ concentrations}

Presentación: 6-7/10/2020

Doctorando:

\section{Emir Saab}

Energías Alternativas, Tecnología y Desarrollo Sustentable (EnAlTecS), Centro de Investigación y Desarrollo en Ciencia y Tecnología de Materiales (CITEMA), Facultad Regional La Plata, Universidad Tecnológica Nacional - Argentina emirsaab@gmail.com

\section{Directora:}

\section{Ana Castro Luna}

\section{Co-directores:}

\section{Mariano Asteazarán y Germán Céspedes}

\section{Resumen}

Las celdas de combustible son dispositivos electroquímicos que convierten directamente la energía química proveniente de una reacción en energía eléctrica. La búsqueda de un combustible limpio, sustentable y con alta energía ha llevado a la creciente consideración de combustibles basados en nitrógeno, como el amoníaco.

El amoníaco líquido se puede almacenar en grandes tanques a temperatura ambiente de forma seguro como los actuales combustibles fósiles. Cada año se producen más de 200 millones de toneladas de amoníaco y se distribuyen a nivel mundial a través de tuberías, y camiones cisterna lo que hace que el amoníaco esté disponible y sea barato.

El platino (Pt), es el catalizador por excelencia en las celdas de combustible debido a su alta electroactividad. Sin embargo, este metal precioso no es abundante en la naturaleza y su costo es elevado, lo que provoca que el desarrollo de celdas de combustible no sea comercialmente atractivo (Cespedes et al., 2016). El Departamento de Energía de EE. UU. (DoE) ha estimado que para hacer un uso masivo de las celdas el precio debería reducirse a unos $40 \mathrm{USD} / \mathrm{kW}$ en sistemas de $80 \mathrm{~kW}$ (Qaseem et al., 2016). Para reducir el costo asociado con el uso de Pt se sintetizan catalizadores nanoestructurados, aumentando así en gran medida la superficie específica y el número de átomos que se encuentran en la superficie del catalizador. Además, el Pt se suele combinar con otros elementos para potenciar su actividad catalítica (Zhong et al., 2013). La actividad catalítica de los materiales a preparar depende fuertemente del método de síntesis a implementar, ya que puede controlar el tamaño, forma y composición de las nanopartículas catalíticas. En los últimos años se han desarrollado métodos sintéticos reproducibles. De esta forma, se pueden obtener nanopartículas combinando diferentes metales en forma de aleación. Se evaluaron electrocatalizadores conocidos como el ya mencionado Pt, en su forma comercial Pt-ETEK a distintas concentraciones de amoníaco y bimetálicos del tipo PtM (con $\mathrm{M}=\mathrm{Ni}, \mathrm{Ru}$ ) dispersos sobre un soporte conductor de alta superficie, preparados utilizando métodos de síntesis que implican la reducción química de los precursores metálicos para el catalizador en cuestión.

Palabras clave: celda de combustible, tecnologías del hidrogeno, energías renovables, energías alternativas 


\section{Abstract}

Fuel cells are electrochemical devices that convert directly chemical energy in electrical energy. The search for a clean, economical and sustainable fuel with high density energy has led to the growing consideration of fuels based on nitrogen, such as ammonia.

Ammonia is an excellent source of hydrogen in its liquid form. Liquid ammonia can be stored in large tanks at room temperature and is safer than propane and as safe as gasoline. More than 200 million tons of ammonia are produced each year and distributed globally via pipelines, tankers \& trucks, making ammonia readily available and cheap.

Platinum (Pt) is the catalyst par excellence in fuel cells due to its high electroactivity. However, this precious metal is not abundant in nature and its cost is high, which causes the development of fuel cells is not commercially attractive (Cespedes et al., 2016). The US Department of Energy (DOE) has estimated that to make the mass use of the cells the price should be reduced to about $40 \mathrm{USD} / \mathrm{kW}$ in $80 \mathrm{~kW}$ systems (Qaseem et al., 2016). To reduce the cost associated with the use of Pt are synthesized nanostructured catalysts, thereby greatly increasing the surface area specific and the number of atoms found on the surface of the catalyst. Additionally, the Pt is usually combined with other elements to enhance the catalytic activity thereof (Zhong et al., 2013). The catalytic activity of the materials to be prepared strongly depends on the method of synthesis to be implemented, since it can control the size, shape and composition of the catalytic nanoparticles. In recent years reproducible synthetic methods have been developed. In this way, nanoparticles can be obtained by combining different metals in alloy shape, core-shell, etc.

Electrocatalysts known as the aforementioned Pt, in its commercial form Pt-ETEK to different concentrations of ammonia, and bimetalic of the PtM type (with $\mathrm{M}=\mathrm{Ni}, \mathrm{Ru}$ ) dispersed on a high-surface conductive support were evaluated using synthetic methods that involve the chemical reduction of the metallic precursors for the catalyst in question.

Keywords: fuel cell, hydrogen technology, renewable energy, alternative energy

\section{Introducción}

Para la síntesis de electrocatalizadores empleados en la reacción de oxidación de amoníaco (AOR), el método utilizado, juega un papel muy importante en la performance de los materiales activos producidos. La actividad catalítica de los materiales a preparar depende fuertemente del método de síntesis a implementar, ya que éste puede controlar la composición, grado de aleación, tamaño de partícula, estructura superficial, morfología, forma y composición de las nanopartículas catalíticas (Park et al., 2015; Qaseem et al., 2016; Shao et al., 2016).

Se desarrollaron y evaluaron electrocatalizadores dispersados sobre un soporte conductor de alta área superficial, en este caso carbón VULCAN ${ }^{\circledR}$ empleando un método de síntesis que involucra la reducción química de los precursores metálicos para el catalizador en cuestión, empleando agentes surfactantes para controlar el tamaño y forma de la nanopartícula y como agente reductor etilenglicol (método poliol), borohidruro de sodio, $\mathrm{H}_{2} / \mathrm{N}_{2}$ a altas temperatura, etc. (Asteazaran et al., 2015).

En la determinación de los catalizadores para la electrooxidación de amoniaco en medio alcalino se tuvo en cuenta la composición de los catalizadores deseados, la relación atómica más adecuada entre los elementos que conformarán el catalizador, la estructura y tamaño de las nanopartículas, la carga de material activo sobre el soporte conductor, etc. (Endo et al., 2004; Silva et al., 2015; Zhong et al., 2013).

La evaluación electrocatalítica de los materiales sintetizados para la AOR incluyó una serie de parámetros tales como la Actividad Másica (MA) y la Actividad Específica (SA), la densidad de corriente normalizada por el Área Superficial Electroquímicamente Activa (ECSA), durabilidad, es decir, la habilidad del electrocatalizador a resistir cambios permanentes de la performance en el tiempo (Katayama et al., 2015; Zhong et al., 2013). Los catalizadores sintetizados, fueron producidos por un método de impregnación modificada (Asteazaran, 2018). Estos materiales se han estudiado como potenciales catalizadores para la electrooxidación del amoniaco y se emplearon técnicas como voltamperometría cíclica (CV) y cronoamperometría (CA). 


\section{Desarrollo}

El platino es el catalizador más activo para el proceso de oxidación de amoníaco, sin embargo, es caro y se inactiva fácilmente por la adsorción de nitrógeno. Se han investigado catalizadores mono y bimetálicos. Para hacer que las celdas de combustible de amoníaco sean comercialmente atractivas, la cantidad de metales preciosos debe reducirse. Esto se puede lograr utilizando electrocatalizadores como nanopartículas dispersas en soportes conductores de alta área de superficie, por ejemplo, negro de carbón VULCAN ${ }^{\circledR}$.

Se sintetizaron catalizadores bimetálicos soportados sobre dicho carbón, tales como PtRu y PtNi utilizando un método de impregnación modificado (Asteazaran et al., 2015; Asteazaran, 2018). Estos materiales sintetizados han sido estudiados como potenciales catalizadores para la electrooxidación de amoníaco.

Se utilizaron técnicas como CV y CA para la caracterización electroquímica. Estos estudios para la AOR se llevaron a cabo en una celda de PTFE de tres electrodos, empleando como electrodo de trabajo un electrodo de disco rotante (RDE) de carbón vítreo $\left(0,07 \mathrm{~cm}^{2}\right)$, sobre el que se depositó una capa delgada de catalizador, una gran superficie de Pt (chapa de Pt de $1 \mathrm{~cm}^{2}$ ) como contraelectrodo y un electrodo de $\mathrm{Ag} / \mathrm{AgCl}$ como electrodo de referencia $(\mathrm{E}=0,22 \mathrm{~V})$. Se utilizó $\mathrm{KOH} 1 \mathrm{M}$ como electrolito soporte, al que se agregaron cantidades crecientes de $\mathrm{NH}_{4} \mathrm{OH}$ y las mediciones electroquímicas se llevaron a cabo a temperatura ambiente, previa desoxigenación con $\mathrm{N}_{2}$ de la solución electrolítica, utilizando un potenciostato Gamry Reference $3000^{\circledR}$.

Para la preparación del electrodo de trabajo se pulió el carbón vítreo con una suspensión de alúmina y se limpió de forma adecuada. Se utilizó agua ultra pura obtenida de un sistema Milli-Q ${ }^{\circledR}$ en todos los procedimientos experimentales. La tinta catalítica se preparó dispersando el polvo del electrocatalizador en agua. La misma se ultrasonicó durante 30 min. Posteriormente, se depositaron $12 \mu \mathrm{l}$ del dispersado sobre la superficie del RDE y una vez seco se adicionaron una micro cantidad de solución de Nafion® al $5 \%$ en metanol. Se empleó como referencia el comportamiento de un catalizador comercial de Pt E-TEK ${ }^{\circledR}$.

Se realizaron CV a una velocidad de barrido de $20 \mathrm{mVs}^{-1}$ en $\mathrm{KOH} 1 \mathrm{M}$, como electrolito soporte, y concentraciones crecientes de 0,05 a 0,5 $\mathrm{M}$ de $\mathrm{NH}_{4} \mathrm{OH}$. Los resultados para los diferentes catalizadores se muestran en las Figura 1, Figura 2 y Figura 3, para Pt-ETEK, PtNi y PtRu, respectivamente. Los electrocatalizadores fueron previamente ciclados en $\mathrm{KOH} 1 \mathrm{M}$ hasta obtener un perfil estable. Los experimentos crono-amperométricos se llevaron a cabo durante $600 \mathrm{~s}$ a $\mathrm{E}=0,35 \mathrm{~V}$ (Figura 4).Todos los gráficos fueron normalizados respecto a la carga de Pt. 


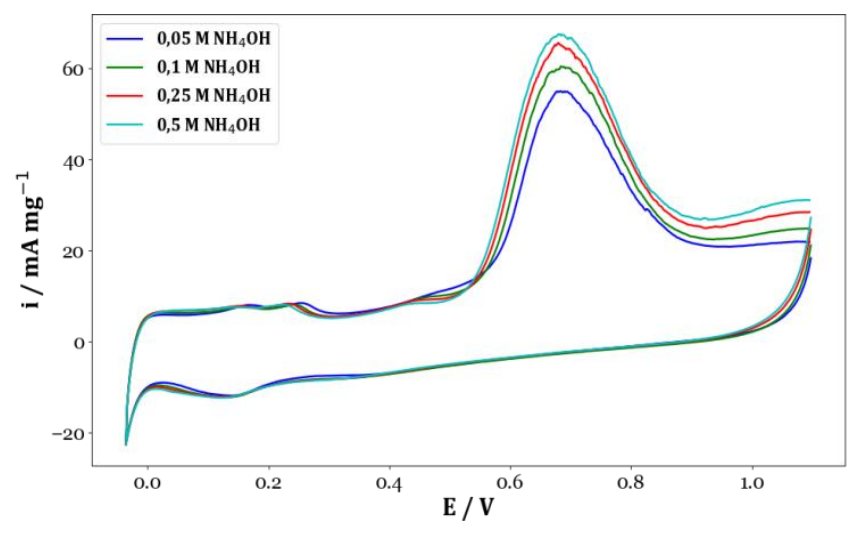

Figura 1: CV a $20 \mathrm{mVs}^{-1}$ del Pt-ETEK a diferentes concentraciones de $\mathrm{NH}_{4} \mathrm{OH}$

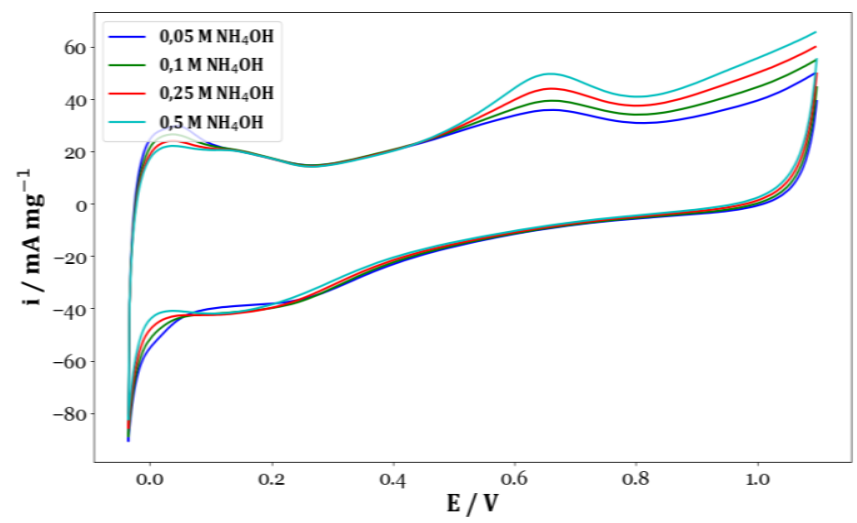

Figura 3: CV a $20 \mathrm{mVs}-1$ del PtRu a diferentes concentraciones de $\mathrm{NH}_{4} \mathrm{OH}$

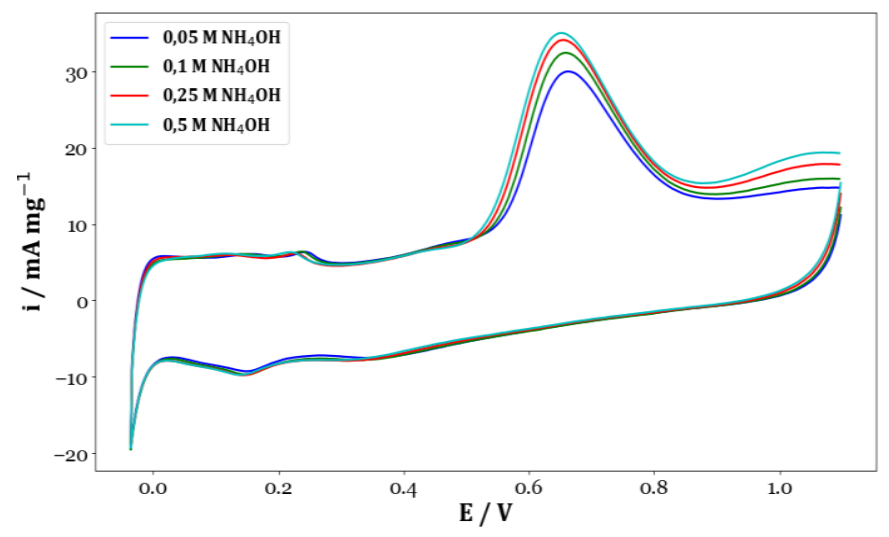

Figura 2: CV a $20 \mathrm{mVs}-1$ del PtNi a diferentes concentraciones de $\mathrm{NH}_{4} \mathrm{OH}$

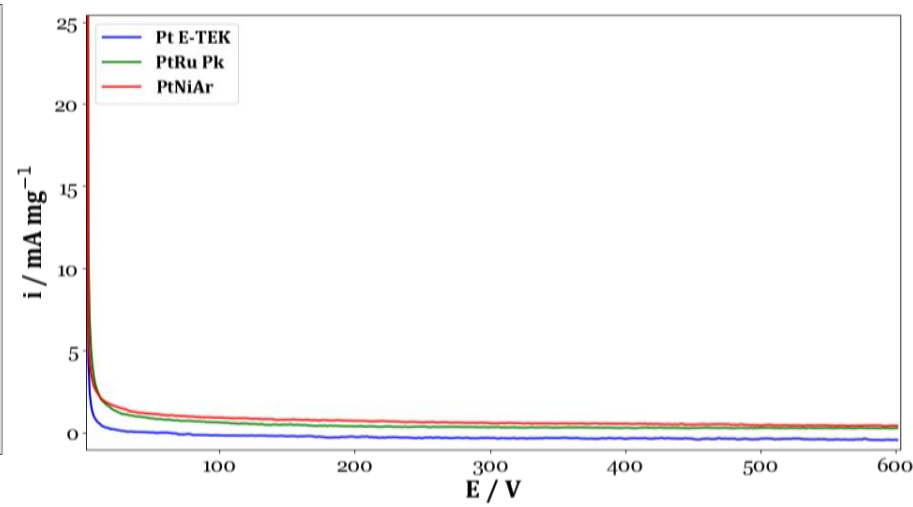

Figura 4: CA a E=0.35 V de Pt-ETEK, PtRu, PtNi durante $600 \mathrm{~s}$ a una concentración de $\mathrm{NH}_{4} \mathrm{OH} 0.5 \mathrm{M}$

\section{Conclusiones}

Se logró sintetizar de forma adecuada materiales nanoestructurados con buenas propiedades electrocatalíticas frente a la AOR en medio alcalino. El método de impregnación modificado ha mostrado ser muy adecuado para la obtención de este tipo de material de electrodo para la celda de combustible de amoniaco directa. De los materiales sintetizados, el PtRu, resultó ser el material menos activo para electrooxidar amoniaco. Así también, se observa que una concentración de $\mathrm{NH}_{4} \mathrm{OH} 0,5 \mathrm{M}$ es muy apropiada para la evaluación de la AOR ya que se aprecia una pequeña diferencia entre las concentraciones más altas de amoníaco. 


\section{Referencias}

Asteazaran, M. (2018). Preparación y caracterización de materiales catalíticos para una Celda de Combustible de Metanol Directo. Análisis del desempeño de un prototipo. (Tesis doctoral). Universidad Tecnologica Nacional 409.

Asteazaran, M., Cespedes, G., Moreno, M. S., Bengió, S., \& Castro Luna, A. M. (2015). Searching for suitable catalysts for a passive direct methanol fuel cell cathode. International Journal of Hydrogen Energy, 40(42), 14632-14639. https://doi.org/10.1016/j.ijhydene.2015.05.134

German Cespedes, Mariano Asteazaran, \& Ana M. Castro Luna. (2016). Effect of Water Content in the Gas Diffusion Layer of H2/O2 PEM Fuel Cell. Journal of Materials Science and Engineering A, 6(4). https://doi.org/10.17265/2161-6213/2016.7-8.004

Endo, K., Nakamura, K., Katayama, Y., \& Miura, T. (2004). Pt-Me (Me = Ir, Ru, Ni) binary alloys as an ammonia oxidation anode. Electrochimica Acta, 2503-2509. https://doi.org/10.1016/j.electacta.2004.01.025

Katayama, Y., Okanishi, T., Muroyama, H., Matsui, T., \& Eguchi, K. (2015). Electrochemical Oxidation of Ammonia over Rare Earth Oxide Modified Platinum Catalysts. The Journal of Physical Chemistry C, 119(17), 9134-9141. https://doi.org/10.1021/acs.jpcc.5b01710

Park, S.-A., Lee, E.-K., Song, H., \& Kim, Y.-T. (2015). Bifunctional enhancement of oxygen reduction reaction activity on Ag catalysts due to water activation on LaMnO3 supports in alkaline media. Scientific Reports, 5(1), 13552. https://doi.org/10.1038/srep13552

Qaseem, A., Chen, F., Wu, X., \& Johnston, R. L. (2016). Pt-free silver nanoalloy electrocatalysts for oxygen reduction reaction in alkaline media. Catalysis Science \& Technology, 6(10), 3317-3340. https://doi.org/10.1039/C5CY02270C

Shao, M., Chang, Q., Dodelet, J.-P., \& Chenitz, R. (2016). Recent Advances in Electrocatalysts for Oxygen Reduction Reaction. Chemical Reviews, 116(6), 3594-3657. https://doi.org/10.1021/acs.chemrev.5b00462

Silva, J. C. M., da Silva, S. G., De Souza, R. F. B., Buzzo, G. S., Spinacé, E. V., Neto, A. O., \& Assumpção, M. H. M. T. (2015). PtAu/C electrocatalysts as anodes for direct ammonia fuel cell. Applied Catalysis A: General, 490, 133-138. https://doi.org/10.1016/j.apcata.2014.11.015

Zhong, C., Hu, W. B., \& Cheng, Y. F. (2013). Recent advances in electrocatalysts for electro-oxidation of ammonia. J. Mater. Chem. A, 1(10), 3216-3238. https://doi.org/10.1039/C2TA00607C 